\title{
D. CRISP
}

\section{W. MORAN}

\section{A. Pollington \\ P. SHIUE}

\section{Substitution invariant cutting sequences}

\author{
Journal de Théorie des Nombres de Bordeaux, tome 5, $\mathrm{n}^{\circ} 1$ (1993), \\ p. $123-137$ \\ <http://www.numdam.org/item?id=JTNB_1993_5_1_123_0>
}

(C) Université Bordeaux 1, 1993, tous droits réservés.

L'accès aux archives de la revue "Journal de Théorie des Nombres de Bordeaux » (http://jtnb.cedram.org/) implique l'accord avec les conditions générales d'utilisation (http://www.numdam.org/conditions). Toute utilisation commerciale ou impression systématique est constitutive d'une infraction pénale. Toute copie ou impression de ce fichier doit contenir la présente mention de copyright.

\section{Numdam}

Article numérisé dans le cadre du programme

Numérisation de documents anciens mathématiques

http://www.numdam.org/ 
Journal de Théorie des Nombres

de Bordeaux 5 (1993), 123-137

\title{
Substitution invariant cutting sequences
}

\author{
by D. Crisp, W. Moran, A. Pollington and P. Shiue
}

\section{Introduction}

This work arose from a problem discussed in an article by Tom Brown, [1]. He considers the sequences

$$
f_{\alpha}(n)=[(n+1) \alpha]-[n \alpha]-[\alpha],
$$

$n \geq 1$, where $\alpha$ is a positive irrational and $[x]$ is the greatest integer not exceeding $x$. It is clear that $f_{\alpha}=f_{\alpha+1}$ and so without loss of generality we assume $0<\alpha<1$, in which case,

$$
f_{\alpha}(n)=[(n+1) \alpha]-[n \alpha] .
$$

There is a large literature on these sequences (see for example [3] and [6]). Each sequence $f_{\alpha}$ consists of 0 's and 1's only and we can, without ambiguity, omit the commas. A substitution $W$ for such a sequence is a pair of maps

$$
W: 0 \rightarrow W_{0}, 1 \rightarrow W_{1},
$$

where $W_{0}$ and $W_{1}$ are finite strings of 0 's and 1's. The result of applying this substitution to $f_{\alpha}$ is the sequence of 0 's and 1's obtained by replacing each 0 in $f_{\alpha}$ by $W_{0}$ and each 1 by $W_{1}$, we denote it by $W\left(f_{\alpha}\right)$. Brown shows that if $1 / \alpha$ has a purely periodic continued fraction expansion, then there is a non-trivial substitution, $W$, leaving $f_{\alpha}$ invariant, that is, $W\left(f_{\alpha}\right)=f_{\alpha}$, and he describes $W$. He also demonstrates an $\alpha$ (for which $1 / \alpha$ does not have a purely periodic continued fraction expansion) with the property that $f_{\alpha}$ is fixed only by the trivial substitution.

We show that the obvious conjecture is not entirely true. We do this by giving a complete characterisation of those $\alpha$ for which $f_{\alpha}$ is invariant under some non-trivial substitution. We also give a full description of the substitutions concerned. 
Ito and Yasutomi, [4], have also considered substitution invariance for the sequences $f_{\alpha}$ but with $n \geq 0$ instead of $n \geq 1$. They obtain a result similar to Brown's. Because they include the extra term $f_{\alpha}(0)=0$, the substitutions which arise in their work are different to both Brown's and our's. Ito and Yasutomi point out that their substitutions may be viewed as automorphisms of the free group generated by 0 and 1 . (The same is true of Brown's and of our's.) In this context we also mention the work of H. Cohn, [2], where, automorphisms of the free group of rank two and the invariance of certain sequences under substitutions are discussed.

Our approach is to reformulate the problem in terms of the cutting sequences of lines in the plane. A precise definition of cutting sequences and some of their properties will be given in the next section, $\S 2$. Their connection with the sequences $f_{\alpha}$ is described in the theorem at the end of that section. Our main theorem for substitution invariant cutting sequences is proved in section $\S 3$. The translation of that theorem into a corresponding one for sequences of the form $f_{\alpha}$ together with a discussion of the relationship between our work and that of Brown and also of Ito and Yasutomi is provided in the final section, $\S 4$.

\section{Cutting Sequences}

The cutting sequence of a general line in the plane is described by Series, [5]. Here however, we only consider lines of the form $y=\beta x$ with $\beta$ a positive irrational and we restrict our attention to the positive quadrant. The cutting sequences of such lines are defined by the following procedure. Construct, in the first quadrant of the plane, the square grid consisting of all vertical and horizontal lines through integer points. Label the intersections of $y=\beta x$ with the grid using 0 if the grid line crossed is vertical and 1 if it is horizontal. The sequence of labels, read from the origin out, is called the cutting sequence of $y=\beta x$ and is denoted here by $\mathbf{S}_{\beta}$. In describing these sequences we shall use the standard abbreviation $0^{k}$ for a block of $k$ consecutive 0 's. The expression $1^{k}$ will be interpreted similarly. When $k=0$ the empty block is meant.

We will consider the effect of certain substitutions on the sequences $\mathbf{S}_{\beta}$ and hence establish some of their properties. First, however, we note that $\beta$ is uniquely determined by $\mathbf{S}_{\beta}$, that is, if $\gamma$ is another positive irrational and $\mathbf{S}_{\gamma}=\mathbf{S}_{\beta}$ then $\gamma=\beta$. To see this, observe that if $\gamma \neq \beta$ then there are points of self-intersection of the grid lying between the lines $y=\gamma x$ and $y=\beta x$ and hence $\mathbf{S}_{\gamma} \neq \mathbf{S}_{\beta}$.

Let $R$ denote the substitution

$$
R: 0 \rightarrow 1,1 \rightarrow 0
$$


Since reflection in $y=x$ interchanges the lines $y=\beta x$ and $y=(1 / \beta) x$ and also the horizontal and vertical lines of the grid, it can be seen that

$$
R\left(\mathbf{S}_{\beta}\right)=\mathbf{S}_{1 / \beta}
$$

If $0<\beta<1$ then $1 / \beta>1$ and it follows that we can, if desired, use $R$ to deduce the properties of the cutting sequences of lines with slope less than 1 from those of lines with slope greater than 1 .

For $k \geq 0$ let $G_{k}$ denote the substitution

$$
G_{k}: 0 \rightarrow 1^{k} 0,1 \rightarrow 1
$$

We claim that

$$
G_{k}\left(\mathbf{S}_{\beta}\right)=\mathbf{S}_{\beta+k} .
$$

In order to prove this, we allow the vertical grid lines to partition $y=\beta x$ into segments. Specifically, set $P_{n}=(n, n \beta)$ and for $n \geq 0$ denote the segment of $y=\beta x$ from $P_{n}$ up to $P_{n+1}$, including $P_{n+1}$ but excluding $P_{n}$, by $L_{n}$. Evidently the block of $\mathbf{S}_{\beta}$ corresponding to the segment $L_{n}$ is $1^{i} 0$ where $i=[(n+1) \beta]-[n \beta]$. Set $\beta^{\prime}=\beta+k$ and similarly partition the line $y=\beta^{\prime} x$ into segments $L_{n}^{\prime}$, so that, the block of $\mathbf{S}_{\beta^{\prime}}$ corresponding to $L_{n}^{\prime}$ is $1^{j} 0$ where $j=\left[(n+1) \beta^{\prime}\right]-\left[n \beta^{\prime}\right]$. Since $\beta^{\prime}=\beta+k$, we know $j=i+k$ and thus $1^{j} 0=G_{k}\left(1^{i} 0\right)$. The truth of the claim is now clear. We remark that the linear transformation $(x, y) \mapsto(x, y+k x)$ transforms $L_{n}$ into $L_{n}^{\prime}$ and $y=\beta x$ into $y=\beta^{\prime} x$.

We have just seen that $\mathbf{S}_{\beta}$ can be partitioned into blocks of the form $1^{i} 0$ where $i=[(n+1) \beta]-[n \beta]$. Since the only possible values for $i$ are $b$ and $b+1$ where $b=[\beta]$, the only possible blocks occurring in such a partitioning are $1^{b} 0$ and $1^{(b+1)} 0$. Because $\beta$ is irrational both must occur. Further, if $n=0$ then $i=b$ and so $\mathbf{S}_{\beta}$ starts with $1^{b} 0$.

For $k \geq 0$ let the substitution $H_{k}$ be defined by $H_{k}=G_{k} \circ R$, so that,

$$
H_{k}: 0 \rightarrow 1,1 \rightarrow 1^{k} 0
$$

The sequence $\mathbf{S}_{\beta}$ can also be partitioned into the blocks 1 and $1^{b} 0$ and therefore is the result of applying $H_{b}$ to some other sequence of 0 's and 1's. By using $H_{b}=G_{b} \circ R$ and the properties of $G_{b}$ and $R$ described above, it is not hard to verify that in fact

$$
\mathbf{S}_{\beta}=H_{b}\left(\mathbf{S}_{\beta^{\prime}}\right)
$$


where $\beta^{\prime}=1 /(\beta-b)$. We can now repeat the argument starting with $\mathbf{S}_{\beta^{\prime}}$ and so on. The resulting algorithm is best understood by expanding $\beta$ as a continued fraction, say

$$
\beta=\left[b_{0}, b_{1}, b_{2}, \ldots\right],
$$

where $b_{0}=b \geq 0$ and $b_{1}, b_{2}, b_{3}, \ldots$ are positive integers. If for $i \geq 0$ we define

$$
\beta_{i}=\left[b_{i}, b_{i+1}, b_{i+2}, \ldots\right],
$$

then $b_{i}=\left[\beta_{i}\right]$ and $\beta_{i+1}=1 /\left(\beta_{i}-b_{i}\right)$ and so $\mathbf{S}_{\beta_{i}}=H_{b_{i}}\left(\mathbf{S}_{\beta_{i+1}}\right)$. By induction we obtain

$$
\mathbf{S}_{\beta}=H_{b_{0}} \circ H_{b_{1}} \circ \cdots \circ H_{b_{i}}\left(\mathbf{S}_{\beta_{i+1}}\right) .
$$

As $i$ increases the strings defining the composition $H_{b_{0}} \circ H_{b_{1}} \circ \cdots \circ H_{b_{i}}$ lengthen and our description of $\mathbf{S}_{\beta}$ improves. While it is not necessary for the purposes of this paper, we point out that this expansion leads to an expression for $\mathbf{S}_{\beta}$ as the limit as $i$ increases to infinity of the strings

$$
H_{b_{0}} \circ H_{b_{1}} \circ \cdots \circ H_{b_{i}}(1) \text {. }
$$

The following theorem describes the connection between the sequences $\mathbf{S}_{\beta}$ and the sequences $f_{\alpha}$. In its proof (and that of the lemma in section $\S 3$ ) we will use a cancellative property of the substitutions $R, G_{k}$ and $H_{k}$. Specifically, if $\mathbf{S}$ and $\mathbf{S}^{\prime}$ are any sequences of 0 's and 1's and $W$ one of $R$, $G_{k}$ or $H_{k}$ then $W(\mathbf{S})=W\left(\mathbf{S}^{\prime}\right)$ if and only if $\mathbf{S}=\mathbf{S}^{\prime}$.

Theorem 1. Let $\alpha$ and $\beta$ be irrationals with $0<\alpha<1$ and $\beta>0$ then $f_{\alpha}=\mathbf{S}_{\beta}$ if and only if

$$
\alpha=\frac{1}{(1 / \beta+1)} \text {. }
$$

Proof. Consider the line $y=\alpha x$ and its cutting sequence $\mathbf{S}_{\alpha}$. As before, let the vertical grid lines partition $y=\alpha x$ into segments. That is, set $P_{n}=(n, n \alpha)$ and for $n \geq 0$ let $L_{n}$ denote the segment from $P_{n}$ up to $P_{n+1}$, including $P_{n+1}$ but excluding $P_{n}$. The block in $\mathbf{S}_{\alpha}$ corresponding to $L_{n}$ is $1^{i} 0$ where

$$
i=[(n+1) \alpha]-[n \alpha]=f_{\alpha}(n) .
$$

This block is 0 if $f_{\alpha}(n)=0$ and 10 if $f_{\alpha}(n)=1$. Clearly, applying the substitution

$$
W: 0 \rightarrow 0,1 \rightarrow 10
$$


to $f_{\alpha}$ yields the cutting sequence $\mathbf{S}_{\alpha}$ of $y=\alpha x$ except for the block due to the segment $L_{0}$. The missing block is 0 and thus applying the substitution

$$
V: 0 \rightarrow 0,1 \rightarrow 01
$$

yields the entire cutting sequence, that is, $V\left(f_{\alpha}\right)=\mathbf{S}_{\alpha}$. We can now deduce that, $f_{\alpha}=\mathbf{S}_{\beta}$ if and only if $\mathbf{S}_{\alpha}=V\left(\mathbf{S}_{\beta}\right)$. The substitution $V$ is equal to $R \circ H_{1}$ and so

$$
V\left(\mathbf{S}_{\beta}\right)=R \circ H_{1}\left(\mathbf{S}_{\beta}\right)=R\left(\mathbf{S}_{1 / \beta+1}\right)=\mathbf{S}_{\gamma},
$$

where $\gamma=1 /(1 / \beta+1)$. It follows that $f_{\alpha}=\mathbf{S}_{\beta}$ if and only if $\mathbf{S}_{\alpha}=\mathbf{S}_{\gamma}$ but $\mathbf{S}_{\alpha}=\mathbf{S}_{\gamma}$ if and only if $\alpha=\gamma$ and the proof is complete.

\section{Substitution Invariant Cutting Sequences}

Theorem 1 shows that Brown's problem is solved if we can characterise those $\beta$ for which there is a substitution leaving $\mathbf{S}_{\beta}$ invariant. In order to do that we require the lemma immediately below. Following the lemma we state and prove our main theorem. It contains the characterisation sought.

Lemma. Let $\beta>1$ and $\gamma>1$ be irrational, say $\beta=\left[b_{0}, b_{1}, b_{2}, \ldots\right]$ and $\gamma=\left[c_{0}, c_{1}, c_{2}, \ldots\right]$. If there is a substitution $W$ such that $W\left(\mathbf{S}_{\beta}\right)=\mathbf{S}_{\gamma}$, then, either,

(1) $c_{0} \geq b_{0}, W=G_{c_{0}-b_{0}}$ and $\beta-b_{0}=\gamma-c_{0}$, or,

(2) there exists a substitution $W^{\prime}$ such that $W=H_{c_{0}} \circ W^{\prime}$ and further, $W^{\prime}\left(\mathbf{S}_{\beta}\right)=\mathbf{S}_{\gamma_{1}}$ where $\gamma_{1}=\left[c_{1}, c_{2}, c_{3}, \ldots\right]$.

Proof. Let $\beta_{1}=\left[b_{1}, b_{2}, b_{3}, \ldots\right]>1, \gamma_{1}=\left[c_{1}, c_{2}, c_{3}, \ldots\right]>1$ and $\gamma_{2}=$ $\left[c_{2}, c_{3}, c_{4}, \ldots\right]>1$, so that, $\mathbf{S}_{\beta}=H_{b_{0}}\left(\mathbf{S}_{\beta_{1}}\right), \mathbf{S}_{\gamma}=H_{c_{0}}\left(\mathbf{S}_{\gamma_{1}}\right)$ and $\mathbf{S}_{\gamma_{1}}=$ $H_{c_{1}}\left(\mathbf{S}_{\gamma_{2}}\right)$ and suppose $W\left(\mathbf{S}_{\beta}\right)=\mathbf{S}_{\gamma}$ where $W$ is the substitution

$$
W: 0 \rightarrow W_{0}, 1 \rightarrow W_{1} .
$$

The two possible outcomes in the lemma depend on the two possible forms of $W_{1}$.

Case (1): The string $W_{1}$ does not contain a 0 . In this case we will show that $c_{0} \geq b_{0}$ and $W=G_{c_{0}-b_{0}}$. It will then follow that

$$
\mathbf{S}_{\gamma}=W\left(\mathbf{S}_{\beta}\right)=G_{c_{0}-b_{0}}\left(\mathbf{S}_{\beta}\right)=\mathbf{S}_{\beta+c_{0}-b_{0}}
$$

and hence $\gamma=\beta+c_{0}-b_{0}$ implying that statement (1) of the lemma is true. 
If $W_{1}$ does not contain a 0 then it is of the form $1^{i}$ for some $i \geq 1$ (the case $i=0$ clearly does not arise). By counting the number of 1 's between 0 's in $\mathbf{S}_{\gamma}$ we will show that, in fact, $i=1$. First note that $W_{0}$ contains a zero, so that we can write $W_{0}=1^{j} 0 X_{0} 1^{k}$ where $j$ and $k$ are non-negative integers and $X_{0}$ is some block of 0 's and 1's which is either empty or ends with a 0 . We know $\mathbf{S}_{\beta}$ starts with $1^{b_{0}} 0$ and contains the block $01^{\left(b_{0}+1\right)} 0$, thus, $\mathbf{S}_{\gamma}$ starts with $W_{1}^{b_{0}} W_{0}$ and contains $W_{0} W_{1}^{\left(b_{0}+1\right)} W_{0}$. On substituting the expressions for $W_{0}$ and $W_{1}$ we find that $\mathbf{S}_{\gamma}$ starts with $1^{\left(i b_{0}+j\right)} 0$ and contains $01^{\left(k+i\left(b_{0}+1\right)+j\right)} 0$. Thus $i b_{0}+j=c_{0}$ and $k+i\left(b_{0}+1\right)+j=c_{0}+1$, which can only happen if $i=1, k=0$ and $j=c_{0}-b_{0}$, so that,

$$
W: 0 \rightarrow 1^{\left(c_{0}-b_{0}\right)} 0 X_{0}, 1 \rightarrow 1
$$

To see that $W=G_{c_{0}-b_{0}}$ it remains to show that $X_{0}$ is empty. We suppose not and obtain a contradiction. Since $X_{0}$ occurs immediately after a 0 in $\mathbf{S}_{\gamma}$ every 0 in it is preceded by $1^{c_{0}}$ at least. It follows that $X_{0}=H_{c_{0}}\left(X_{0}^{\prime}\right)$ for some string $X_{0}^{\prime}$. Let $V$ be the substitution

$$
V: 0 \rightarrow 0,1 \rightarrow 1 X_{0}^{\prime}
$$

and note that $H_{c_{0}} \circ V=W \circ H_{b_{0}}$ and thus

$$
H_{c_{0}} \circ V\left(\mathbf{S}_{\beta_{1}}\right)=W \circ H_{b_{0}}\left(\mathbf{S}_{\beta_{1}}\right)=W\left(\mathbf{S}_{\beta}\right)=\mathbf{S}_{\gamma}=H_{c_{0}}\left(\mathbf{S}_{\gamma_{1}}\right)
$$

It follows that $V\left(\mathbf{S}_{\beta_{1}}\right)=\mathbf{S}_{\gamma_{1}}$. We use this last equality to examine the form of $X_{0}^{\prime}$. By counting the number of 1's between 0 's in $\mathbf{S}_{\gamma_{1}}$, we see that $X_{0}^{\prime}$ contains a 0 (by assumption $X_{0}^{\prime}$ is non-empty) and we can write $X_{0}^{\prime}=1^{i} 0 Y_{0} 1^{j}$ where $i$ and $j$ are non-negative integers and $Y_{0}$ is some block which is either empty or ends with a 0 . We know $\mathbf{S}_{\beta_{1}}$ starts with a 1 and that 110 occurs. Therefore, $\mathbf{S}_{\gamma_{1}}$ starts with $1 X_{0}^{\prime}$ and $1 X_{0}^{\prime} 1 X_{0}^{\prime} 0$ occurs. On substituting $X_{0}^{\prime}=1^{i} 0 Y_{0} 1^{j}$ we find that $\mathbf{S}_{\gamma_{1}}$ starts with $1^{(i+1)} 0$ and that $01^{(j+1+i)} 0$ and $01^{j} 0$ both occur. It follows that $i+1=c_{1}$ and $j+1+i$ and $j$ are either $c_{1}$ or $c_{1}+1$. The only possibility is that $c_{1}=1, i=0$ and $j=1$. Thus $X_{0}^{\prime}=0 Y_{0} 1$ and

$$
V: 0 \rightarrow 0,1 \rightarrow 10 Y_{0} 1
$$

Since $c_{1}=1$ and $Y_{0}$ occurs immediately after a 0 in $\mathbf{S}_{\gamma_{1}}$, there is some (possibly empty) string $Y_{0}^{\prime}$ such that $Y_{0}=H_{1}\left(Y_{0}^{\prime}\right)$. Let $U$ be the substitution

$$
U: 0 \rightarrow 1 Y_{0}^{\prime} 1,1 \rightarrow 1 Y_{0}^{\prime} 0
$$


and observe that $H_{1} \circ U=V \circ G_{1}$ and hence

$$
H_{1} \circ U\left(\mathbf{S}_{\beta_{1}-1}\right)=V \circ G_{1}\left(\mathbf{S}_{\beta_{1}-1}\right)=V\left(\mathbf{S}_{\beta_{1}}\right)=\mathbf{S}_{\gamma_{1}}=H_{1}\left(\mathbf{S}_{\gamma_{2}}\right) .
$$

It follows that $U\left(\mathbf{S}_{\beta_{1}-1}\right)=\mathbf{S}_{\gamma_{2}}$. We can now examine $Y_{0}^{\prime}$. The sequence $\mathbf{S}_{\beta_{1}-1}$ contains $10^{i} 1$ and $10^{(i+1)} 1$ for some $i \geq 0$ and therefore $\mathbf{S}_{\gamma_{2}}$ contains $0\left(1 Y_{0}^{\prime} 1\right)^{i} 1 Y_{0}^{\prime} 0$ and $0\left(1 Y_{0}^{\prime} 1\right)^{(i+1)} 1 Y_{0}^{\prime} 0$. Again, by counting 1 's in these expressions, it is clear that $Y_{0}^{\prime}$ is not empty and in fact contains a zero. Further, since $Y_{0}^{\prime}$ is followed by a 0 in $\mathbf{S}_{\gamma_{2}}$ it must end with $1^{c_{2}}$. But $Y_{0}^{\prime}$ is also followed by $1^{2}$ implying that $1^{\left(c_{2}+2\right)}$ occurs. This contradicts the properties of $\mathbf{S}_{\gamma_{2}}$ and our assumption about $X_{0}$ was not correct. It follows that $X_{0}$ is the empty string and $W=G_{c_{0}-b_{0}}$ as initially indicated.

Case (2): The string $W_{1}$ contains a 0 . In this case we will show that $W_{0}=H_{c_{0}}\left(W_{0}^{\prime}\right)$ for some string $W_{0}^{\prime}$. We already know that $W_{1}=H_{c_{0}}\left(W_{1}^{\prime}\right)$ for some string $W_{1}^{\prime}$ (since $W_{1}$ is the initial segment of $\mathbf{S}_{\gamma}$ and thus every 0 in it is preceded by $1^{c_{0}}$ at least). It will follow then, that $W=H_{c_{0}} \circ W^{\prime}$ where $W^{\prime}$ is the substitution

$$
W^{\prime}: 0 \rightarrow W_{0}^{\prime}, 1 \rightarrow W_{1}^{\prime}
$$

and in this event, since

$$
H_{c_{0}} \circ W^{\prime}\left(\mathbf{S}_{\beta}\right)=W\left(\mathbf{S}_{\beta}\right)=\mathbf{S}_{\gamma}=H_{c_{0}}\left(\mathbf{S}_{\gamma_{1}}\right)
$$

we know $W^{\prime}\left(\mathbf{S}_{\beta}\right)=\mathbf{S}_{\gamma_{1}}$ and statement (2) of the lemma is true.

We will show that $W_{0}=H_{c_{0}}\left(W_{0}^{\prime}\right)$ by supposing otherwise and obtaining a contradiction. If $W_{0}$ is not of the stated form then it must begin with $1^{i} 0$ where $i<c_{0}$. We write $W_{0}=1^{i} 0 X_{0}$ where $0 \leq i<c_{0}$ and $X_{0}$ is possibly empty. We are assuming $W_{1}$ contains a 0 and since it is the initial segment of $\mathbf{S}_{\gamma}$ we can write $W_{1}=1^{c_{0}} 0 X_{1} 1^{j}$ where $j$ is a non-negative integer and $X_{1}$ is either empty or ends with a 0 . The blocks $W_{1} W_{0}$ and $W_{1} W_{1}$ both occur in $\mathbf{S}_{\gamma}$ and thus $01^{(j+i)} 0$ and $01^{\left(j+c_{0}\right)} 0$ do also. This implies $i=c_{0}-1$, $j=1$ and

$$
W: 0 \rightarrow 1^{\left(c_{0}-1\right)} 0 X_{0}, 1 \rightarrow 1^{c_{0}} 0 X_{1} 1
$$

The blocks $X_{0}$ and $X_{1}$ are preceded by 0 's in $\mathbf{S}_{\gamma}$ and thus there exist (possibly empty) $X_{0}^{\prime}$ and $X_{1}^{\prime}$ such that $X_{0}=H_{c_{0}}\left(X_{0}^{\prime}\right)$ and $X_{1}=H_{c_{0}}\left(X_{1}^{\prime}\right)$. Let $V$ be the substitution

$$
V: 0 \rightarrow 1 X_{1}^{\prime} 1 X_{0}^{\prime}, 1 \rightarrow 1 X_{1}^{\prime} 0
$$


so that $H_{c_{0}} \circ V=W \circ G_{1}$ and therefore

$$
H_{c_{0}} \circ V\left(\mathbf{S}_{\beta-1}\right)=W \circ G_{1}\left(\mathbf{S}_{\beta-1}\right)=W\left(\mathbf{S}_{\beta}\right)=\mathbf{S}_{\gamma}=H_{c_{0}}\left(\mathbf{S}_{\gamma_{1}}\right) .
$$

It follows that $V\left(\mathbf{S}_{\beta-1}\right)=\mathbf{S}_{\gamma_{1}}$. We use this last equality to examine $X_{0}^{\prime}$ and $X_{1}^{\prime}$. There are two possibilities for $X_{1}^{\prime}$.

Subcase (1): The string $X_{1}^{\prime}$, contains a 0 . Since $1 X_{1}^{\prime}$ is the initial segment of $\mathbf{S}_{\gamma_{1}}$ and $X_{1}^{\prime}$ occurs in $\mathbf{S}_{\gamma_{1}}$ preceding both a 0 and a 1 , we can write $X_{1}^{\prime}=1^{\left(c_{1}-1\right)} 0 Y_{1} 1^{c_{1}}$ where $Y_{1}$ is either empty or ends with a 0 . Thus the occurrence of $X_{1}^{\prime} 1 X_{0}^{\prime} 1$ in $\mathbf{S}_{\gamma_{1}}$ implies that $X_{0}^{\prime}$ is not empty and in fact begins with 0 . We write $X_{0}^{\prime}=0 Y_{0}$ where $Y_{0}$ is possibly empty. Hence

$$
V: 0 \rightarrow 1^{c_{1}} 0 Y_{1} 1^{\left(c_{1}+1\right)} 0 Y_{0}, 1 \rightarrow 1^{c_{1}} 0 Y_{1} 1^{c_{1}} 0 .
$$

As usual, there exist (possibly empty) $Y_{0}^{\prime}$ and $Y_{1}^{\prime}$ such that $Y_{0}=H_{c_{1}}\left(Y_{0}^{\prime}\right)$ and $Y_{1}=H_{c_{1}}\left(Y_{1}^{\prime}\right)$. The form of $Y_{1}^{\prime}$ may be elicited using the fact that $U\left(\mathbf{S}_{\beta-1}\right)=\mathbf{S}_{\gamma_{2}}$ where

$$
U: 0 \rightarrow 1 Y_{1}^{\prime} 01 Y_{0}^{\prime}, 1 \rightarrow 1 Y_{1}^{\prime} 1 .
$$

That this is true follows from the equalities $H_{c_{1}} \circ U=V$ and $V\left(\mathbf{S}_{\beta-1}\right)=$ $\mathbf{S}_{\gamma_{1}}=H_{c_{1}}\left(\mathbf{S}_{\gamma_{2}}\right)$. Since $\mathbf{S}_{\beta-1}$ contains $01^{i} 0$ and $01^{(i+1)} 0$ for some $i \geq 0$, the sequence $\mathrm{S}_{\gamma_{2}}$ contains $01 Y_{0}^{\prime}\left(1 Y_{1}^{\prime} 1\right)^{i} 1 Y_{1}^{\prime} 0$ and $01 Y_{0}^{\prime}\left(1 Y_{1}^{\prime} 1\right)^{(i+1)} 1 Y_{1}^{\prime} 0$. As before, by counting 1 's between 0 's, it is clear that $Y_{1}^{\prime}$ contains a 0 . Further, since $Y_{1}^{\prime}$ is followed by a 0 and by a 1 in $\mathbf{S}_{\gamma_{2}}$ it must end with $1^{c_{2}}$. But $Y_{1}^{\prime}$ is also followed by $1^{2}$ implying that $1^{\left(c_{2}+2\right)}$ occurs. This is a contradiction and our assumption about $X_{1}^{\prime}$ was not correct.

Subcase (2): The string $X_{1}^{\prime}$ does not contain 0 . As before, we use the fact that $V\left(\mathbf{S}_{\beta-1}\right)=\mathbf{S}_{\gamma_{1}}$ where $V: 0 \rightarrow 1 X_{1}^{\prime} 1 X_{0}^{\prime}, 1 \rightarrow 1 X_{1}^{\prime} 0$ to determine the form of $X_{0}^{\prime}$ and $X_{1}^{\prime}$. Clearly $X_{0}^{\prime}$ is not empty and in fact contains a 0 . We write $X_{0}^{\prime}=1^{i} 0 Y_{0} 1^{j}$ and $X_{1}^{\prime}=1^{k}$ where $i, j$ and $k$ are non-negative integers and $Y_{0}$ is either empty or ends with a 0 . Note that $Y_{0}=H_{c_{1}}\left(Y_{0}^{\prime}\right)$ for some (possibly empty) $Y_{0}^{\prime}$. Assume for the moment that $\beta-1 \geq 1$, that is, $b_{0} \geq 2$. In this case, $\mathbf{S}_{\gamma_{1}}$ starts with $1 X_{1}^{\prime} 0$ and both $01 X_{1}^{\prime} 1 X_{0}^{\prime}$ and $X_{0}^{\prime} 1 X_{1}^{\prime} 0$ occur. On substituting the expressions for $X_{0}^{\prime}$ and $X_{1}^{\prime}$ we find that $\mathrm{S}_{\gamma_{1}}$ starts with $1^{(1+k)} 0$ and that both $01^{(k+2+i)} 0$ and $01^{(j+1+k)} 0$ occur. The only possibility is that $k+1=c_{1}, i=0$ and $j=0$ or 1 . We have shown that $X_{0}^{\prime}=0 Y_{0} 1^{j}$ and $X_{1}^{\prime}=1^{\left(c_{1}-1\right)}$ and so

$$
V: 0 \rightarrow 1^{\left(c_{1}+1\right)} 0 Y_{0} 1^{j}, 1 \rightarrow 1^{c_{1}} 0
$$


where $j$ is 0 or 1 . We now claim that the result of the substitution

$$
U: 0 \rightarrow 01 Y_{0}^{\prime} 0^{j}, 1 \rightarrow 1
$$

in $\mathbf{S}_{\beta-1}$ is $\mathbf{S}_{\gamma_{2}}$. Again this can be verified by checking that $H_{c_{1}} \circ U=V$. The equation $U\left(\mathbf{S}_{\beta-1}\right)=\mathbf{S}_{\gamma_{2}}$ and the form of $U$ satisfy the hypothesis of case (1) and hence $U=G_{c_{2}-\left(b_{0}-1\right)}$. This is clearly impossible and our assumption that $\beta-1 \geq 1$ was wrong. Assume now that $\beta-1<1$, so that, $b_{0}=1$ and $\beta_{1}=1 /(\beta-1)$. Since $R\left(\mathbf{S}_{\beta_{1}}\right)=\mathbf{S}_{\beta-1}$ we know that $V \circ R\left(\mathbf{S}_{\beta_{1}}\right)=\mathbf{S}_{\gamma_{1}}$. Clearly then, $\mathbf{S}_{\gamma_{1}}$ starts with $1 X_{1}^{\prime} 1 X_{0}^{\prime}$ and both $X_{0}^{\prime} 1 X_{1}^{\prime} 1 X_{0}^{\prime}$ and $X_{0}^{\prime} 1 X_{1}^{\prime} 0$ occur. On substituting the expressions for $X_{0}^{\prime}$ and $X_{1}^{\prime}$ we find that $\mathbf{S}_{\gamma_{1}}$ starts with $1^{(k+2+i)} 0$ and that both $01^{(j+k+2+i)} 0$ and $01^{(j+1+k)} 0$ occur. The only possibility is that $k+2=c_{1}, i=0$ and $j=1$, that is, $X_{0}^{\prime}=0 Y_{0} 1$ and $X_{1}^{\prime}=1^{\left(c_{1}-2\right)}$, so that,

$$
V: 0 \rightarrow 1^{c_{1}} 0 Y_{0} 1,1 \rightarrow 1^{\left(c_{1}-1\right)} 0 \text {. }
$$

Let $U$ be the substitution

$$
U: 0 \rightarrow 1 Y_{0}^{\prime} 1,1 \rightarrow 1 Y_{0}^{\prime} 0
$$

and note that $H_{c_{1}} \circ U=V \circ R \circ G_{1}$ and thus

$$
H_{c_{1}} \circ U\left(\mathbf{S}_{\beta_{1}-1}\right)=V \circ R \circ G_{1}\left(\mathbf{S}_{\beta_{1}-1}\right)=V\left(\mathbf{S}_{\beta-1}\right)=\mathbf{S}_{\gamma_{1}}=H_{c_{1}}\left(\mathbf{S}_{\gamma_{2}}\right) \text {. }
$$

It follows that $U\left(\mathbf{S}_{\beta_{1}-1}\right)=\mathbf{S}_{\gamma_{2}}$. However, we have already shown in the final argument of case (1) that this situtation leads to a contradiction. Again, we conclude that our assumption about $X_{1}^{\prime}$ was not valid.

There are no more possibilities and the proof of the lemma is complete.

\section{ThEOREM 2.}

(1) Let $\beta>1$ be irrational. The cutting sequence $\mathbf{S}_{\beta}$ of the line $y=\beta x$ is invariant under some non-trivial substitution $W$ if and only if $\beta$ has a continued fraction expansion of the form $\beta=\left[b_{0}, \overline{b_{1}, \ldots, b_{n}}\right]$ where $b_{n} \geq b_{0} \geq 1$. Further, if that is the case and $n$ is minimal then $W$ must be a power of

$$
H_{b_{0}} \circ H_{b_{1}} \circ \cdots \circ H_{b_{n-1}} \circ G_{b_{n}-b_{0}}
$$

and all such substitutions leave $\mathbf{S}_{\beta}$ invariant.

(2) Let $0<\beta<1$ be irrational. The cutting sequence $\mathbf{S}_{\beta}$ of the line $y=\beta x$ is invariant under some non-trivial substitution $W$ 
if and only if $\beta$ has a continued fraction expansion of the form $\beta=\left[0, b_{0}, \overline{b_{1}, \ldots, b_{n}}\right]$ where $b_{n} \geq b_{0}$. Further, if that is the case and $n$ is minimal then $W$ must be a power of

$$
R \circ H_{b_{0}} \circ H_{b_{1}} \circ \cdots \circ H_{b_{n-1}} \circ G_{b_{n}-b_{0}} \circ R
$$

and all such substitutions leave $\mathbf{S}_{\beta}$ invariant.

Proof. Because the substitution $R$ interchanges the sequences $\mathbf{S}_{\beta}$ and $\mathbf{S}_{1 / \beta}$, part (2) of the theorem is an easy consequence of part (1). We give our full attention to part (1).

We start with the reverse implication, that is, given $\beta=\left[b_{0}, \overline{b_{1}, \ldots, b_{n}}\right]$ where $b_{n} \geq b_{0}$ we will show that $\mathbf{S}_{\beta}$ is invariant under the non-trivial substitution

$$
W=H_{b_{0}} \circ H_{b_{1}} \circ \cdots \circ H_{b_{n-1}} \circ G_{b_{n}-b_{0}} .
$$

By direct calculation, $W\left(\mathbf{S}_{\beta}\right)=\mathbf{S}_{\gamma}$ where $\gamma=\left[b_{0}, b_{1}, \ldots, b_{n-1}, \beta-b_{0}+b_{n}\right]$. We are given that $\beta=\left[b_{0}, \overline{b_{1}, \ldots, b_{n}}\right]$ and therefore $\beta=\gamma$ and $W$ fixes $\mathbf{S}_{\beta}$, as required.

Next we prove the forward implication, that is, given that $\mathbf{S}_{\beta}$ is invariant under some non-trivial substitution $W$ we will show that $\beta=\left[b_{0}, \overline{b_{1}, \ldots, b_{n}}\right]$ where $b_{n} \geq b_{0} \geq 1$. We write $\beta=\left[b_{0}, b_{1}, b_{2}, \ldots\right]$. Since $W\left(\mathbf{S}_{\beta}\right)=\mathbf{S}_{\beta}$, the lemma applies and thus, either, $W=G_{0}$, or, there exists a substitution $W^{\prime}$ such that $W=H_{b_{0}} \circ W^{\prime}$ and $W^{\prime}\left(\mathbf{S}_{\beta}\right)=\mathbf{S}_{\beta_{1}}$ where $\beta_{1}=\left[b_{1}, b_{2}, b_{3}, \ldots,\right]$. Because $W$ is non-trivial, the latter must be true. A further application of the lemma to $W^{\prime}\left(\mathbf{S}_{\beta}\right)=\mathbf{S}_{\beta_{1}}$ implies that, either,

(1) $b_{1} \geq b_{0}, W^{\prime}=G_{b_{1}-b_{0}}$ and $\beta-b_{0}=\beta_{1}-b_{1}$, or,

(2) $W^{\prime}=H_{b_{1}} \circ W^{\prime \prime}$ for some $W^{\prime \prime}$ and $W^{\prime \prime}\left(\mathbf{S}_{\beta}\right)=\mathbf{S}_{\beta_{2}}$ where $\beta_{2}=$ $\left[b_{2}, b_{3}, b_{4}, \ldots,\right]$.

If condition (1) is true, we are finished since we have shown that $b_{1} \geq b_{0}$, $W=H_{b_{0}} \circ G_{b_{1}-b_{0}}$ and $\beta=\left[b_{0}, \overline{b_{1}}\right]$. If condition (2) is true, the lemma can be applied again to $W^{\prime \prime}\left(\mathbf{S}_{\beta}\right)=\mathbf{S}_{\beta_{2}}$ and so on.

Repeated applications of the lemma must eventually lead to condition (1) because each application strictly reduces the combined length of the strings defining the relevant substitution. Let the total number of applications be $n+1 \geq 2$. From the arguments above it is clear that $b_{n} \geq b_{0}$ and

$$
W=H_{b_{0}} \circ H_{b_{1}} \circ \cdots \circ H_{b_{n-1}} \circ G_{b_{n}-b_{0}} .
$$


Again, direct calculation shows $W\left(\mathbf{S}_{\beta}\right)=\mathbf{S}_{\gamma}$ where

$$
\gamma=\left[b_{0}, b_{1}, \ldots, b_{n-1}, \beta-b_{0}+b_{n}\right]
$$

By assumption $W\left(\mathbf{S}_{\beta}\right)=\mathbf{S}_{\beta}$ and so $\gamma=\beta$ and $\beta=\left[b_{0}, \overline{b_{1}, \ldots, b_{n}}\right]$.

The first statement in part (1) is now proven and we deal with the second. Suppose $\beta=\left[b_{0}, \overline{b_{1}, \ldots, b_{n}}\right]$ where $b_{n} \geq b_{0} \geq 1$ and that $V$ is a non-trivial substitution fixing $\mathbf{S}_{\beta}$. Further suppose $n$ is minimal. We will show that $V=W^{k}$ for some $k \geq 1$ where

$$
W=H_{b_{0}} \circ H_{b_{1}} \circ \cdots \circ H_{b_{n-1}} \circ G_{b_{n}-b_{0}} .
$$

(By $W^{k}$ we mean the composition of $k$ copies of $W$ ). From the proof of the first statement we know that $\beta=\left[b_{0}, \overline{b_{1}, \ldots, b_{m}}\right]$ where $b_{m} \geq b_{0}$ and that

$$
V=H_{b_{0}} \circ H_{b_{1}} \circ \cdots \circ H_{b_{m-1}} \circ G_{b_{m}-b_{0}} .
$$

Since $n$ is minimal there is some $k \geq 1$ such that $m=k n$. Thus

$$
V=H_{b_{0}} \circ\left(H_{b_{1}} \circ \cdots \circ H_{b_{n}}\right)^{k-1} \circ H_{b_{1}} \circ \cdots \circ H_{b_{n-1}} \circ G_{b_{n}-b_{0}} .
$$

But $H_{b_{n}}=G_{b_{n}-b_{0}} \circ H_{b_{0}}$ and so $V=W^{k}$, as claimed. That all such substitutions fix $\mathbf{S}_{\beta}$ is trivial and the proof is complete.

\section{Substitution invariance for the sequences $f_{\alpha}$}

Using Theorem 1, we can express our results for the sequences $\mathbf{S}_{\beta}$ in terms of the sequences $f_{\alpha}$. The mathematics is not complicated and we obtain the following theorem.

THEOREM 3.

(1) Let $1 / 2<\alpha<1$ be irrational. The sequence $f_{\alpha}$ is invariant under some non-trivial substitution $W$ if and only if $\alpha$ has a continued fraction expansion of the form $\alpha=\left[0,1, a_{2}, \overline{a_{3}, \ldots, a_{n}}\right]$ where $a_{n} \geq a_{2}$. Further, if that is the case and $n$ is minimal then $W$ must be a power of

$$
H_{a_{2}} \circ H_{a_{3}} \circ \cdots \circ H_{a_{n-1}} \circ G_{a_{n}-a_{2}}
$$

and all such substitutions leave $f_{\alpha}$ invariant.

(2) Let $0<\alpha<1 / 2$ be irrational. The sequence $f_{\alpha}$ is invariant under some non-trivial substitution $W$ if and only if $\alpha$ has a continued fraction expansion of the form $\alpha=\left[0, a_{1}, \overline{a_{2}, \ldots, a_{n}}\right]$ where 
$a_{n}+1 \geq a_{1} \geq 2$. Further, if that is the case and $n$ is minimal then $W$ must be a power of

$$
R \circ H_{a_{1}-1} \circ H_{a_{2}} \circ \cdots \circ H_{a_{n-1}} \circ G_{a_{n}+1-a_{1}} \circ R
$$

and all such substitutions leave $f_{\alpha}$ invariant.

Observe that if $\alpha=\left[0,1, a_{2}, \overline{a_{3}, \ldots, a_{n}}\right]$ where $a_{n} \geq a_{2}$ then $1-\alpha=$ $\left[0, a_{2}+1, \overline{a_{3}, \ldots, a_{n}}\right]$ where $a_{n}+1 \geq a_{2}+1 \geq 2$ and vice versa. This type of relation is to be expected when it is noticed that applying $R$ interchanges $f_{\alpha}$ and $f_{1-\alpha}$.

Next, we compare our results with those of Brown. We start with his Theorem 1. It states that, if $\alpha=\left[0, \overline{a_{1}, \ldots, a_{m}}\right]$ then $f_{\alpha}$ is invariant under the substitution

$$
W=h_{a_{1}} \circ h_{a_{2}} \circ \cdots \circ h_{a_{m}}
$$

where, for $k \geq 1$, the substitution $h_{k}$ is defined by

$$
h_{k}: 0 \rightarrow 0^{(k-1)} 1,1 \rightarrow 0^{(k-1)} 10 .
$$

An easy calculation shows $h_{k}=R \circ H_{k-1} \circ H_{1}$ and since $H_{1} \circ R \circ H_{k-1}=H_{k}$ we have

$$
W=R \circ H_{a_{1}-1} \circ H_{a_{2}} \circ H_{a_{3}} \circ \cdots \circ H_{a_{m}} \circ H_{1} \text {. }
$$

There are two possibilities for $a_{1}$, namely, $a_{1}=1$ and $a_{1} \geq 2$. If $a_{1}=1$ then $\alpha=\left[0,1, a_{2}, \overline{a_{3}, \ldots, a_{m}, 1, a_{2}}\right]$ and by part (1) of our theorem, $f_{\alpha}$ is fixed by the substitution

$$
V=H_{a_{2}} \circ \cdots \circ H_{a_{m}} \circ H_{1} \circ G_{0}
$$

Since $G_{0}$ is trivial and so is $R \circ H_{a_{1}-1}$ when $a_{1}=1$, we find, as expected, that $V=W$. If $a_{1} \geq 2$ then $\alpha=\left[0, a_{1}, \overline{a_{2}, \ldots, a_{m}, a_{1}}\right]$ and by part (2) of our theorem, $f_{\alpha}$ is fixed by the substitution

$$
V=R \circ H_{a_{1}-1} \circ H_{a_{2}} \circ \cdots \circ H_{a_{m}} \circ G_{1} \circ R
$$

Again $\dot{V}=W$, this time because $H_{1}=G_{1} \circ R$. The theorems are clearly consistent.

Brown also shows that when $\alpha=[0,5,1,1,1, \ldots]$ there are no non-trivial substitutions fixing $f_{\alpha}$. This is evident from part (2) of our theorem. The remainder of Brown's paper focuses on general quadratic irrationals and substitutions of the form $s \rightarrow C_{1}, t \rightarrow C_{2}$, where $s$ and $t$ are finite strings 
of 0 's and 1's and $C_{1}$ and $C_{2}$ are finite strings of $s$ 's and $t$ 's. While such things are not dealt with here, we remark that our methods can also be applied to them.

We conclude with a discussion of the relevant results in the paper by Ito and Yasutomi. As mentioned Ito and Yasutomi consider substitution invariance for the sequences $f_{\alpha}(n)$ where $n=0$ is allowed. Since the initial term is $f_{\alpha}(0)=0$, we denote these sequences in the natural manner by $0 f_{\alpha}$. For $0<\alpha<1$ they define a sequence $\pi(\alpha)$ of 0 's and 1's and show that if $\alpha$ is irrational with continued fraction expansion, say $\alpha=\left[0, a_{1}, a_{2}, a_{3}, \ldots\right]$, then

$$
\pi(\alpha)=0^{\left(a_{1}-1\right)} 1^{a_{2}} 0^{a_{3}} 1^{a_{4}} 0^{a_{5}} \ldots
$$

They also introduce the substitutions

$$
\gamma_{0}: 0 \rightarrow 0,1 \rightarrow 01
$$

and

$$
\gamma_{1}: 0 \rightarrow 01,1 \rightarrow 1 .
$$

Observe that $\gamma_{0}=R \circ H_{1}$ but no such relation exists for $\gamma_{1}$. Their main result on substitution invariance (for the sequences $0 f_{\alpha}$ ) is contained in Theorem 2.4. While a slightly weaker result is actually stated, it is apparent from their work that if $\pi(\alpha)$ is periodic with minimal period, say

$$
0^{\left(a_{1}-1\right)} 1^{a_{2}} 0^{a_{3}} \ldots 1^{a_{m}} 0^{a},
$$

then $0 f_{\alpha}$ is fixed by the substitution

$$
W=\gamma_{0}^{\left(a_{1}-1\right)} \circ \gamma_{1}^{a_{2}} \circ \gamma_{0}^{a_{3}} \circ \cdots \circ \gamma_{1}^{a_{m}} \circ \gamma_{0}^{a} .
$$

Again we are abbreviating the composition of $i$ copies of a substitution $\gamma$ by $\gamma^{i}$. The connection between this result and ours may be found by noting that if $\mathbf{S}$ and $\mathbf{S}^{\prime}$ are any. two sequences of 0 's and 1's then $\gamma_{0}(0 \mathbf{S})=0 \mathbf{S}^{\prime}$ if and only if $R \circ H_{1}(\mathbf{S})=\mathbf{S}^{\prime}$ and $\gamma_{1}(0 \mathbf{S})=0 \mathbf{S}^{\prime}$ if and only if $G_{1}(\mathbf{S})=\mathbf{S}^{\prime}$. It follows that, if $W=\gamma_{0}^{\left(a_{1}-1\right)} \circ \gamma_{1}^{a_{2}} \circ \gamma_{0}^{a_{3}} \circ \cdots \circ \gamma_{1}^{a_{m}} \circ \gamma_{0}^{a}$, then, $W\left(0 f_{\alpha}\right)=0 f_{\alpha}$ if and only if $V\left(f_{\alpha}\right)=f_{\alpha}$ where

$$
\begin{aligned}
V & =\left(R \circ H_{1}\right)^{a_{1}-1} \circ G_{1}^{a_{2}} \circ\left(R \circ H_{1}\right)^{a_{3}} \cdots \circ G_{1}^{a_{m}} \circ\left(R \circ H_{1}\right)^{a} \\
& =R \circ H_{a_{1}-1} \circ H_{a_{2}} \circ \cdots \circ H_{a_{m}} \circ H_{a} .
\end{aligned}
$$

Given that $0^{\left(a_{1}-1\right)} 1^{a_{2}} 0^{a_{3}} \ldots 1^{a_{m}} 0^{a}$ is a period of $\pi(\alpha)$, there are three possibilities, namely, 
(1) $a_{1}=1, a=0$ and $\alpha=\left[0,1, a_{2}, \overline{a_{3}, \ldots, a_{m-1}, a_{m}^{\prime}}\right]$ where $a_{m}^{\prime}=$ $a_{m}+a_{2}>a_{2}$

(2) $a_{1}=1, a \geq 1$ and $\alpha=\left[0,1, a_{2}, \overline{a_{3}, \ldots, a_{m+2}}\right]$ where $a_{m+1}=a$ and $a_{m+2}=a_{2}$,

(3) $a_{1} \geq 2$ and $\alpha=\left[0, a_{1}, \overline{a_{2}, \ldots, a_{m+1}}\right]$ where $a_{m+1}+1=a_{1}+a \geq$ $a_{1} \geq 2$.

It is not hard to verify now that Ito and Yasutomi's work agrees with ours. We stress however, that, the index $m$ is (by definition) even and therefore, although the period of $\pi(\alpha)$ is minimal, the expressions for $\alpha$ just listed may not be. When this occurs, $V=U^{2}$ (where $U$ is the minimal substitution fixing $f_{\alpha}$ ) and $U$ itself has no corresponding $W$.

Ito and Yasutomi have not discussed the question of whether there are other solutions to the equation $W\left(0 f_{\alpha}\right)=0 f_{\alpha}$. It appears that our results for the sequences $f_{\alpha}$ cannot be used to answer this question directly. However, we believe that our methods are applicable (with appropriate modifications) and could be used to show that there are no other solutions. Since $W\left(0 f_{\alpha}\right)=0 f_{\alpha}$ if and only if $R \circ W \circ R\left(1 f_{1-\alpha}\right)=1 f_{1-\alpha}$, an equivalent problem is the solution of the equation $W\left(1 f_{\alpha}\right)=1 f_{\alpha}$. Note that the sequence $1 f_{\alpha}$ is an equally justified extension of $f_{\alpha}$ when $f_{\alpha}$ is viewed as the cutting sequence of a line (the line $y=\beta x$ where $\beta=1 /(1 / \alpha-1)$ to be precise).

In the final section of their paper, Ito and Yasutomi also provide some insight into the question of substitution invariance for inhomogeneous sequences, that is, sequences of the form

$$
f_{\alpha, \beta}(n)=[(n+1) \alpha+\beta]-[n \alpha+\beta] .
$$

Such sequences are of course the cutting sequences of arbitrary lines in the plane. One of the authors (Crisp) of this paper is currently preparing for publication a complete solution to this more general problem. The sequences $0 f_{\alpha}$ and $1 f_{\alpha}$ will appear as special cases.

\section{REFERENCES}

[1] T. C. BROWN, A characterisation of the quadratic irrationals, Canad. Math. Bull. 34 (1991), 36-41.

[2] H. COHN, Some direct limits of primitive homotopy words and of Markoff geodesics, Discontinuous groups and Riemann surfaces, Ann. of Math. Studies No. 79, Princeton Univ. Press, Princeton, N.J., (1974), 81-98. 
[3] A. S. FRAENKEL, Determination of [ $n \theta]$ by its sequence of differences, Canad. Math. Bull. 21 (1978), 441-446.

[4] Sh. ITO and S. YASUTOMI, On continued fractions, substitutions and characteristic sequences $[n x+y]-[(n-1) x+y]$, Japan J. Math. $16(1990), 287-306$.

[5] C. SERIES, The geometry of Markoff numbers, Math. Intelligencer 7 (1985), 20-29.

[6] K. B. STOLARSKY, Beatty sequences, continued fractions, and certain shift operators, Canad. Math. Bull. 19 (1976), 473-482.

D. Crisp, W. Moran

Mathematics Department

Flinders University of South Austrlia

Bedford Park

SA 5042 AUSTRALIE
A. Pollington
Mathematics Department
Talmage Math. / Computer Building
Brigham Young University
Provoh, Utah 84602 U. S. A.
P. Shiue
Mathematics Department
University of Nevada at Las Vegas
4505 Maryland Pkwy
Las Vegas, NV 89154 U. S. A. 